\title{
Phase measuring deflectometry for obtaining 3D shape of specular surface: a review of the state-of-the-art
}

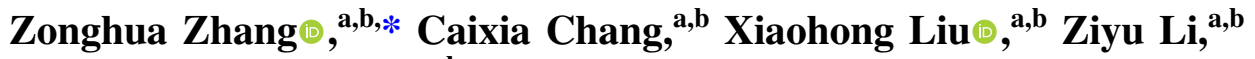 \\ Yanqing Shi, ${ }^{\mathrm{a}, \mathrm{b}}$ Nan Gao, ${ }^{\mathrm{a}}$ and Zhaozong Meng ${ }^{\mathrm{a}}$ \\ ${ }^{a}$ Hebei University of Technology, School of Mechanical Engineering, Tianjin, China \\ ${ }^{b}$ Hebei University of Technology, State Key Laboratory of Reliability and Intelligence of \\ Electrical Equipment, Tianjin, China
}

\begin{abstract}
Phase measuring deflectometry (PMD) is a superior technique to obtain three-dimensional (3D) shape information of specular surfaces because of its advantages of large dynamic range, noncontact operation, full-field measurement, fast acquisition, high precision, and automatic data processing. We review the recent advances on PMD. The basic principle of PMD is introduced following several PMD methods based on fringe reflection. First, a direct PMD (DPMD) method is reviewed for measuring 3D shape of specular objects having discontinuous surfaces. The DPMD method builds the direct relationship between phase and depth data, without gradient integration procedure. Second, an infrared PMD (IR-PMD) method is reviewed to measure specular objects. Because IR light is used as a light source, the IR-PMD method is insensitive to the effect of ambient light on the measured results and has high measurement accuracy. Third, a proposed method is reviewed to measure the 3D shape of partial reflective objects having discontinuous surfaces by combining fringe projection profilometry and DPMD. Then, the effects of error sources that mainly include phase error and geometric calibration error on the measurement results are analyzed, and the performance of the 3D shape measurement system is also evaluated. Finally, the future research directions of PMD are discussed. (C) 2021 Society of Photo-Optical Instrumentation Engineers (SPIE) [DOI: 10.1117/1.OE.60.2.020903]
\end{abstract}

Keywords: 3D shape measurement; phase measuring deflectometry; direct PMD; infrared PMD; specular surface; partial reflective surfaces.

Paper 20201280V received Oct. 27, 2020; accepted for publication Jan. 25, 2021; published online Feb. 17, 2021.

\section{Introduction}

Three-dimensional (3D) shape measurement technique is becoming increasingly important in various applications. ${ }^{1-5}$ Many measurement methods have been put forward to reconstruct the 3D shape of object surfaces, especially for the objects with diffused surfaces. ${ }^{6-10}$ However, the research of 3D shape measurement for specular objects is still in the early stage. It is a challenging problem for measuring 3D shape of specular objects due to the reflective properties of their surfaces. Extensive research efforts have been implemented to increase the measurement accuracy, dynamic range, and speed. ${ }^{11-13}$

Depending on the difference of measurement principles, the 3D shape metrology of measuring specular surfaces can be classified as contact method ${ }^{14}$ and noncontact method. ${ }^{15-28}$ The coordinate measurement machine $(\mathrm{CMM})^{14}$ is a superior choice for measuring specular surfaces by touching the measured surface and scanning along two-dimensional (2D) directions in contact method. Although CMM has the drawbacks of high cost, low speed, and scratching of the surface, ${ }^{29}$ it is still the most widely used method for specular surface measurement because touch probes are highly accurate for dimensional inspection. ${ }^{30,31}$ However, traditional CMMs are limited due to a lack of rotational degree of freedom, so they cannot be used for digitizing complex and free-form surfaces. ${ }^{32}$ Interferometry ${ }^{20-22}$ and deflectometry ${ }^{15-18,23-28}$ are two competitive

*Address all correspondence to Zonghua Zhang, zhzhang@hebut.edu.cn; zhzhangtju@ hotmail.com 
methods among the noncontact measurement methods because of their high measurement accuracy and resolution. Interferometry is a technique using the phenomenon of interference to obtain distance information of simple continuous surfaces, such as planar surfaces and spheres. Since traditional optical interferometry techniques are extremely sensitive to environmental noise, such as mechanical vibration, air turbulence, and temperature drift. The results of 3D shape measurements will be affected by these noises in actual measurements. To tackle this issue, Muhamedsalih et al. ${ }^{33}$ presented a new method that a wavelength scanning interferometer (WSI) is used to measure micro- and nanoscale areal surfaces, which can be immune to environmental noise. In this method, WSI together with an acousto-optic tunable filtering technique is used to measure surfaces with large step heights. Dávila ${ }^{22}$ proposed a sensing method for wavelength scanning interferometry using multiple light sources. With the proposed method, the environmental noise can be successfully reduced and the depth resolution can be improved. However, these methods mentioned above have difficulty in measuring complicated aspheric mirrors or free-form specular objects, such as surfaces with a steep slope and/or large size, because a reference is normally required for interferometry. In recent years, deflectometry has been investigated by many researchers to measure free-form specular objects with a greatly curved and/or large scale surfaces. Phase measuring deflectometry (PMD) is one of the most typical active approaches to obtain the form information of specular surfaces. PMD has been widely studied in recent years because of its advantages of large dynamic range, noncontact operation, full-field measurement, fast acquisition, high precision, and automatic data processing. ${ }^{23-28}$

Zhang et al. ${ }^{34}$ briefly reviewed the advancements on PMD in their recent preliminary work. In this paper, we will review state-of-the-art of PMD in more detail, mainly including the basic principle of PMD, the advancements of PMD, error sources analysis of PMD, challenges, and further development. In Sec. 2, the fundamentals and concepts of PMD are introduced following several PMD methods based on fringe reflection. Section 3 reviews the advancements of PMD. First, a direct PMD (DPMD) method is presented for measuring 3D shape of specular objects having discontinuous surfaces. Then, an infrared PMD (IR-PMD) method is reviewed to measure specular objects. Because IR light is used as a light source, the IR-PMD method is insensitive to the effect of ambient light and has high measurement accuracy. Finally, a proposed method is reviewed to measure the 3D shape of partial reflective objects having discontinuous surfaces by combining fringe projection profilometry (FPP) and DPMD. The following Sec. 4 will analyze the effects of error sources, including the influence of phase error (which caused by the nonlinear influence of imaging and projecting system, display screen, random phase error, and sampling phase error), geometric calibration error on the measurement results and evaluate the performance of the 3D shape measurement system. Challenges and further developments of PMD are briefly reviewed in Sec. 5. The conclusive remarks are provided in Sec. 6.

\section{Fundamental Principle of PMD}

The fundamental principle of PMD depends on the law of light reflection. Figure 1 shows the main devices of a general PMD measurement system, which consists of a digital camera, a liquid crystal display (LCD) screen, a specular surface, and a computer monitor. The LCD screen is equipped to display the phase-shifting sinusoidal fringe patterns generated by the computer. The tested specular object is properly positioned opposite to the LCD screen to reflect the incident lights, following that the deformed fringe patterns modulated by the target surface can be simultaneously captured by an imaging device from a different viewpoint. Then, phase data are calculated from the deformed fringe patterns. After system calibration, the slope data of specular surfaces are obtained using phase information. Finally, 3D global shape of the target surface is reconstructed by integrating the slope data. ${ }^{16,18,35-37}$

Figure 2 shows the schematic diagram of a general PMD measurement system. A reference surface shown in green line is assumed to be parallel to the displaying screen. $h$ is the height of a measured point, and the angle change of the corresponding point relative to the reference plane is $\theta$. Here, $\theta$ also represents slope. There are many possible height and slope combinations to explain the phase point observed by a camera probe ray. As shown in Fig. 2, if there is no height 


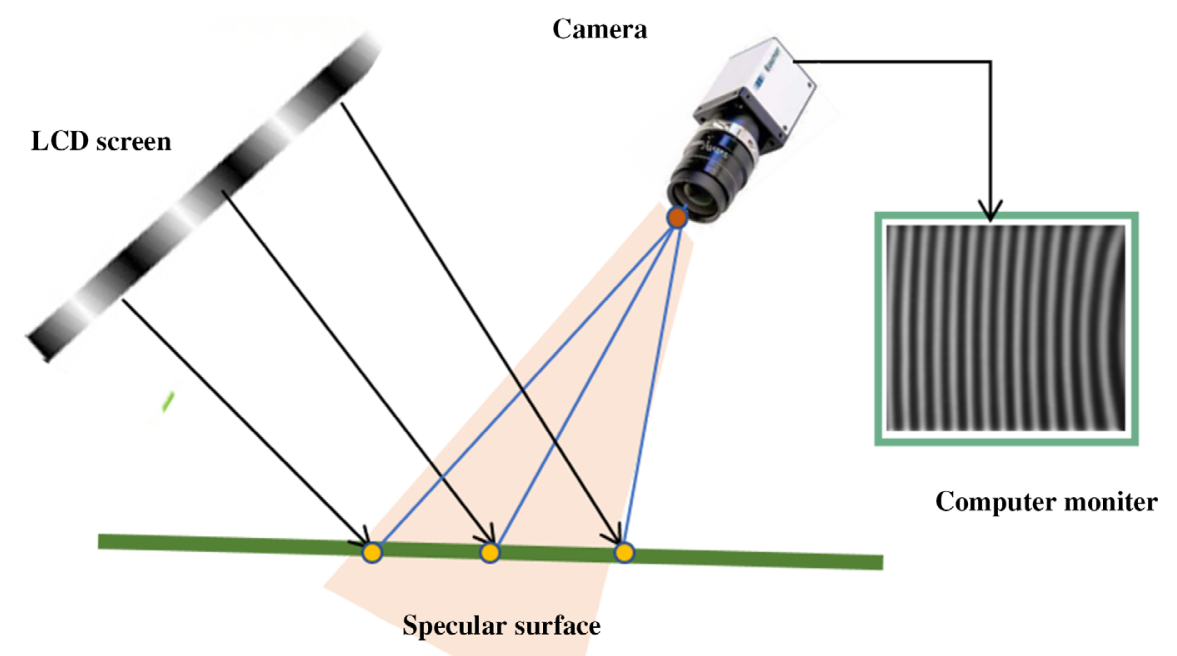

Fig. 1 Measurement principle of a general PMD measurement system.

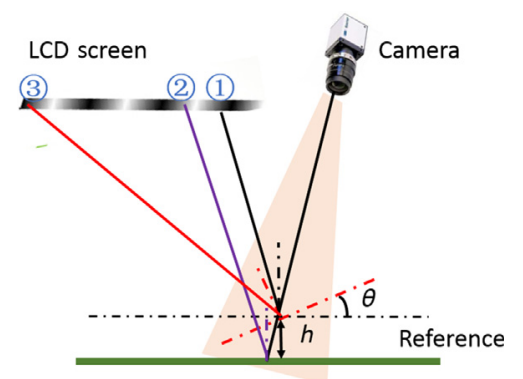

Fig. 2 Schematic diagram of a general PMD measurement system.

and slope change of the measured target surface, the pixel value of the fringe patterns at position (1) is imaged on the CCD plane. If it only has a height change, the pixel value of the fringe patterns at position (2) is imaged at the same position on the CCD plane. If the height and slope both change, the pixel value of the fringe patterns at position (3) is also imaged at the same position on the CCD plane. Therefore, the traditional PMD methods cannot obtain height data directly from phase information.

\subsection{Establishments of PMD}

In general, the traditional PMD separately displays and captures two directional sinusoidal fringe patterns to obtain two components of local surface gradient, which is a procedure that reduces the measurement speed. Liu et al. proposed a technique by displaying cross fringe patterns on the LCD screen to make PMD be possible to be implemented by a one-dimensional translation of the fringe pattern, instead of the common $2 \mathrm{D}$ translation. ${ }^{18}$ However, each point of the reconstructed shape depends on the slopes of surrounding points owing to the integration procedure, and the regularity of the surface must be carefully considered. ${ }^{35-37}$ An integration step in traditional PMD does not necessarily accumulate random errors but often eliminates measurement noise. However, those PMD-based methods mentioned above are only suitable for measuring the specular objects having continuous surfaces due to the procedure of gradient integration. In order to overcome the challenges in classical PMD, several different kinds of regularization approaches with corresponding setups for different requirements in actual measurement are proposed. ${ }^{38-48}$ Some typical PMD establishments with additional components (such as screens or cameras) are shown in Fig. 3. Figure 3(a) shows a measurement method that the LCD screen 


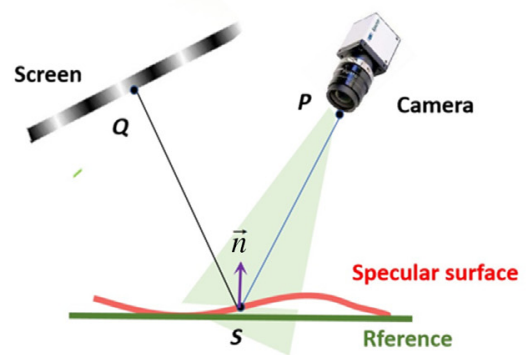

(a)

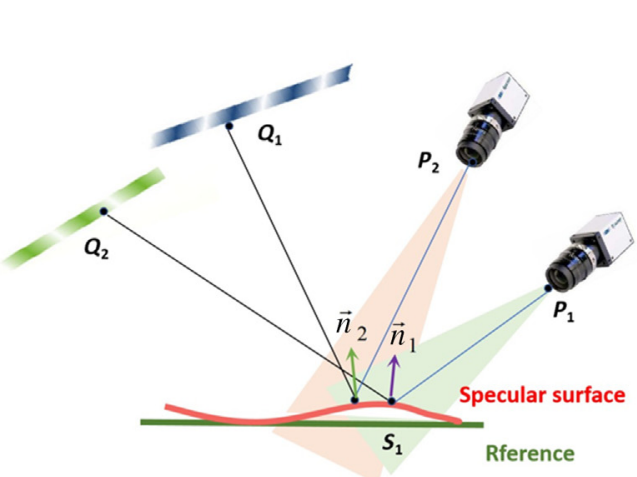

(c)

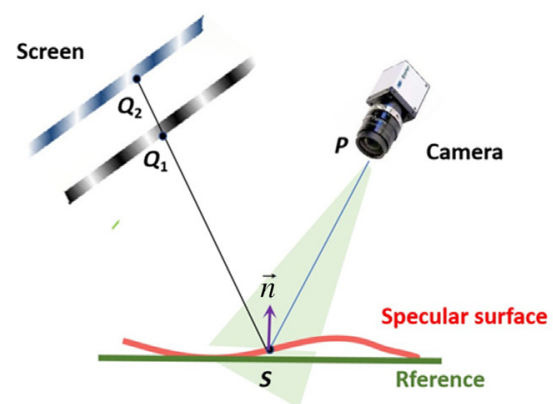

(b)

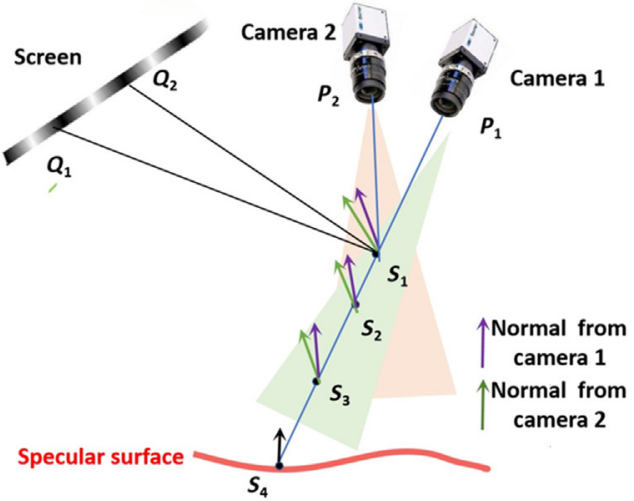

(d)

Fig. 3 Some typical PMD establishments. (a) PMD with a shifted screen, (b) PMD with multiscreens, (c) multicamera PMD with multiscreens, and (d) stereoscopic PMD.

was used as a diffusive light source, which can be vertically moved to two or more different positions to eliminate the accumulation of errors caused by gradient integration. ${ }^{38-40}$ However, movement of the LCD screen increases the instability and measurement time of the system. To resolve the ill-posed problems, $\mathrm{Li}$ et al. ${ }^{41}$ presented a simplified PMD system using a transparent screen and an LCD screen, avoiding the movement of the LCD screen. In Li's system, the two screens can be fixed in a relatively free position and the ray of camera can pass through the transparent screen to an LCD screen. A simple model shown in Fig. 3(b) is used to describe PMD method with multiple screens. ${ }^{4-45,49-51}$ The reflected ray can be determined by the two or more points of intersection on the multiscreens. In Fig. 3(c), multiscreens and multicameras can be used to search the height values from the minimized normal vector. Figure 3(d) describes the stereoscopic PMD, ${ }^{46-48}$ a technique based on multiple imaging sensors. In this technique, the normal vectors calculated from the two cameras should be the same for a surface point, then gradient and 3D data of the measured surface can be obtained by matching normal vectors calculated from the two cameras. However, it takes a large amount of time while searching for space points. Similar to our classifications of PMD, Xu et al. ${ }^{52}$ classified those PMD methods into single screen and sensor-based PMD, multiscreen-based PMD, and multisensor-based PMD.

\subsection{Phase Extraction}

Once the fringe patterns are captured, phase of the fringe patterns needs to be extracted using the well-developed fringe analysis algorithms. ${ }^{1,53-65}$ Phase extraction includes phase wrapping and phase unwrapping. The wrapped phase data can be calculated using single-frame methods ${ }^{1,53-55}$ (transform-based methods, including Fourier transform, and wavelet transform) and multipleframe methods ${ }^{56}$ (multiple-step phase-shifting algorithms). The spatial phase unwrapping and temporal phase unwrapping are used to calculate the absolute phase data. ${ }^{53,57-65}$ In PMD systems, the required phase values are absolute phase; therefore, the fringe orders should be consistent for all measurements. 


\section{Advancements of PMD}

To further advance the development of PMD, three new configurations of DPMD ${ }^{49-51}$ IR-PMD (MPMD), ${ }^{66,67}$ and novel method for measuring partial reflective surfaces ${ }^{68}$ have been developed, which will be reviewed in the following three sections, respectively.

\subsection{Direct Phase-Measuring Deflectometry}

DPMD is a PMD technique based on multiple displaying screens, which builds a direct relationship between phase and depth to directly calculate 3D shape of the specular object from the phase data, instead of integrating the local slope data. ${ }^{49-51}$ A measurement system, as shown in Fig. 4, has been developed to obtain the 3D shape of specular objects by displaying the same fringe pattern onto two LCD screens.

Figure 4 shows the schematic diagram of the DPMD method and its corresponding system setup. An experimental result is displayed to illustrate its measurement capability. Specifically, Fig. 4(a) shows the measurement principle. The system setup includes two LCD screens, a beam splitter (BS), and a camera. Among them, the BS is located between LCD1 and LCD2 to make the virtual image LCD1' of LCD1 parallel to LCD2, the camera is placed at a certain angle with LCD2 to capture the deformed fringes reflected by the tested specular surface. Moreover, a reference is used to obtain geometric parameters of the DPMD system, which needs to be adjusted parallel to the two screens. $\Delta d$ and $d$ represent the distance between LCD1' and LCD2, and that between LCD1' and the reference plane, respectively. Two rays of light are displayed and reflected into the CCD camera via the measured surface and the reference. The absolute phases

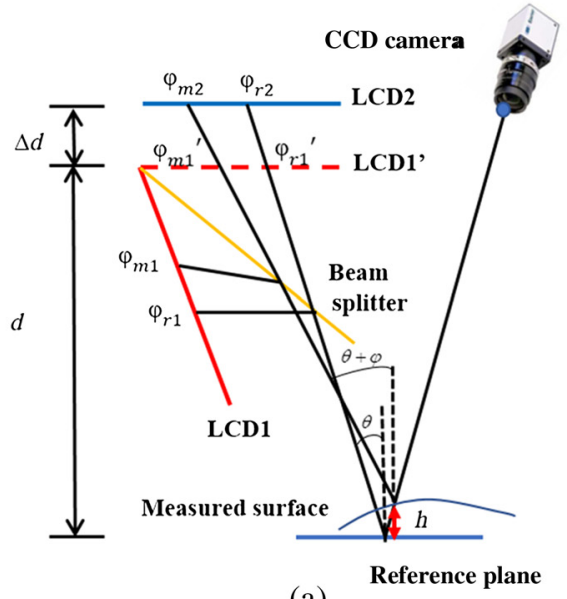

(a)

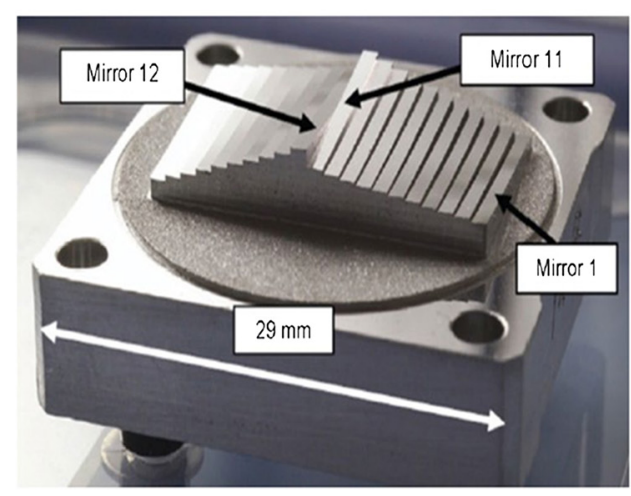

(c)

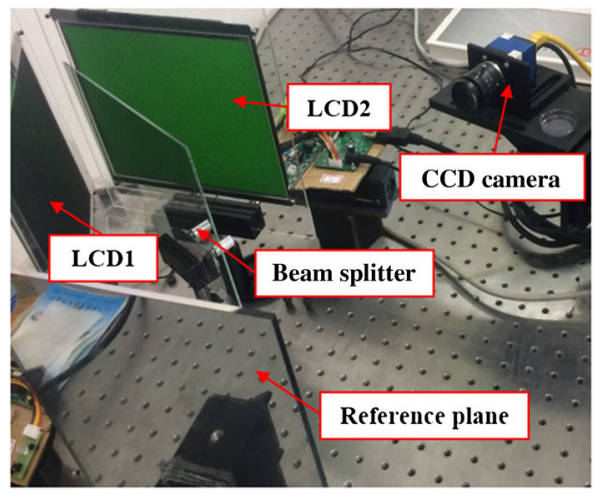

(b)

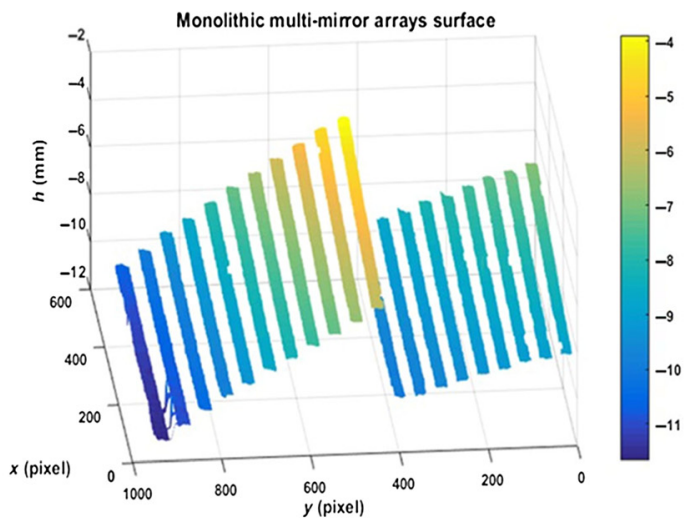

(d)

Fig. 4 Measurement example of DPMD system. ${ }^{49-51}$ (a) Schematic setup of DPMD. (b) Hardware of the experimental setup. (c) Monolithic multimirror array on the MIRI spectrometer optics for the James Webb SpaceTelescope. (d) Measured depth. 
of the two incident rays are denoted $\varphi_{r 1}\left(\operatorname{or} \varphi_{r 1^{\prime}}\right)$ and $\varphi_{r 2}$ on the reference plane and $\varphi_{m 1}$ (or $\left.\varphi_{m 1^{\prime}}\right)$ and $\varphi_{m 2}$ on the measured surface. The angles between the incident ray and the normal vector of the reference and that between the incident ray and the normal vector of the measured surface are $\theta$ and $\theta+\varphi$, respectively. $h$ is the height of the measured specular surface with respect to the reference plane. The hardware system is shown in Fig. 4(b). The MIRI Spectrometer Optics for the James Webb Space Telescope having multiple specular surfaces are measured using the developed DPMD system in Fig. 4(c), and the depth of the measured specular objects shown in Fig. 4(d). Depth data of the measured specular surface can be obtained according to Eq. (1) with calibrated geometric parameters $(\Delta d$ and $d)$ and known phase values $\left(\varphi_{m 1}, \varphi_{m 2}, \varphi_{r 1}\right.$, and $\left.\varphi_{r 2}\right)$ :

$$
h=\frac{\Delta d \cdot\left(\phi_{r 1}-\phi_{m 1}\right)-d \cdot\left[\left(\phi_{r 1}-\phi_{r 2}\right)-\left(\phi_{m 1}-\phi_{m 2}\right)\right]}{\left(\phi_{m 1}-\phi_{m 2}\right)+\left(\phi_{r 1}-\phi_{r 2}\right)} .
$$

Since the depth can be directly reconstructed from the absolute phase without gradient integration, this method can measure specular objects having isolated and/or discontinuous surfaces using the developed DPMD system successfully and accurately.

\subsection{Infrared Phase-Measuring Deflectometry}

The existing PMD methods mostly utilize LCD screens as the structured light source. However, the selection of light source is a key factor to ensure the measurement accuracy of PMD system because visible light is sensitive to ambient light. Therefore, the reconstructed 3D shape data with LCD screen illuminating are mostly affected in actual measurement. To reduce the effects of ambient light on specular surface reconstruction accuracy, Chang et al. ${ }^{66}$ proposed an infraredPMD (IR-PMD) method to measure discontinuous specular components by establishing the direct relationship between absolute phase and depth data, as shown in Fig. 5. Similar to

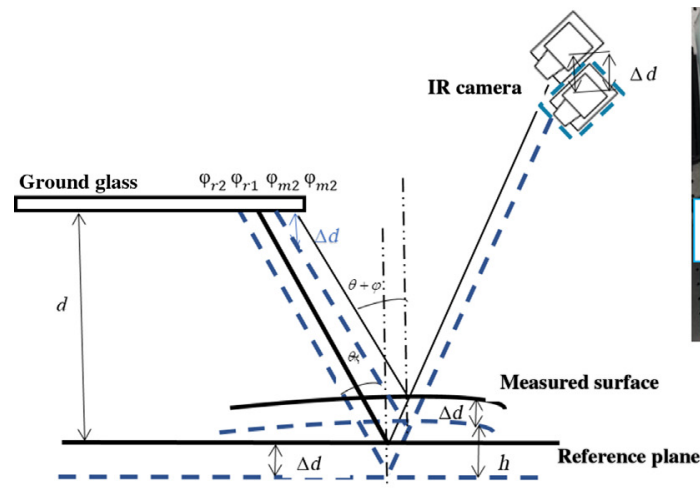

(a)

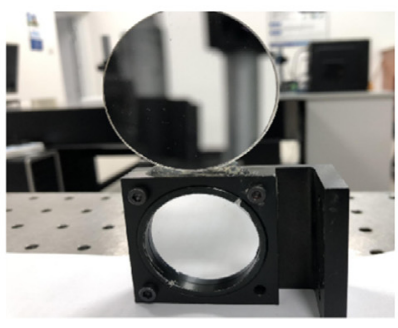

(c)

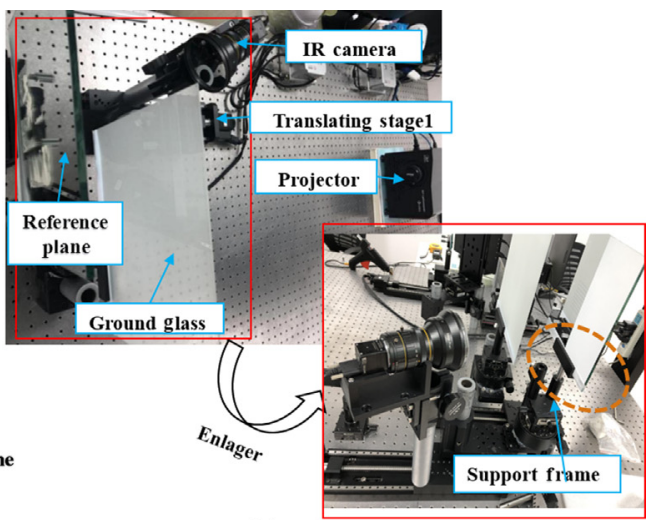

(b)

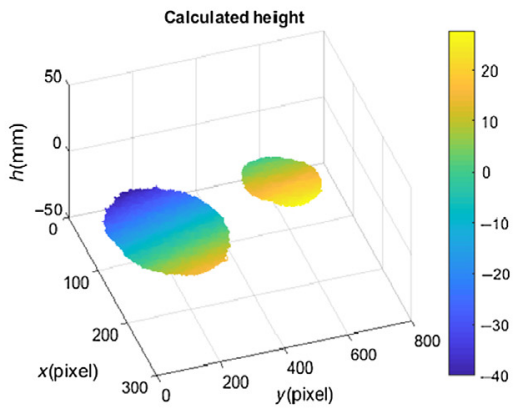

(d)

Fig. 5 Measurement example of IR-PMD. ${ }^{66}$ (a) Principle of IR-PMD. (b) Hardware of the experimental setup. (c) Two separated specular objects. (d) 3D shape data of the measured specular objects. 
DPMD, IR-PMD worked on reconstructing height from the obtained phase data, instead of just integrating the slopes. The proposed system applies IR projector as a light source so that the effects of ambient light can be reduced. In the IR-PMD system, a projector projecting IR sinusoidal fringe patterns onto a ground glass is regarded as an IR digital screen, and an IR camera with the measured specular surfaces is moved simultaneously to two positions by a translating stage to realize two screens design. Moreover, a new geometric calibration method is proposed in Chang's paper using fringe projection and fringe reflection technologies. Figure 5(a) shows the schematic diagram of the IR-PMD system, a mirror being parallel to the ground glass has been chosen as a reference. Just like DPMD, a mathematical model also needs to be derived to directly relate phase to depth. Sinusoidal fringe patterns having the optimum fringe numbers are displayed on the ground glass, which can be regarded as an IR digital screen. The IR camera captures those deformed fringe patterns from two positions of the IR digital screen through the measured specular surfaces. A standard four-step phase-shifting algorithm and an optimum three-fringe number selection method are used to calculate the wrapped and the absolute phase data, respectively. According to Eq. (1), 3D shape data can be directly calculated with the unwrapped phase data calculated from the deformed patterns captured at two positions. Figure 5(b) shows the hardware of the experimental setup. The red line frame shows a partial enlargement of the device, and the orange dashed line circle indicates the mirror whose surface was sprayed in part. Using the proposed IR-PMD system, the authors successfully measured the 3D shape of two separated specular objects shown in Figs. 5(c) and 5(d). The results validate the effectiveness and accuracy of the proposed method.

However, the IR-PMD method mentioned above needs to move the measured objects during the experimental measurement procedure, which will cause random errors. In order to avoid the systematic errors during the movement of the measured objects, a new improved IR-PMD 3D shape measurement method has been presented, as shown in Fig. 6. This method exploits an IR digital display's movement to substitute the camera and the measured object's movement. ${ }^{67}$

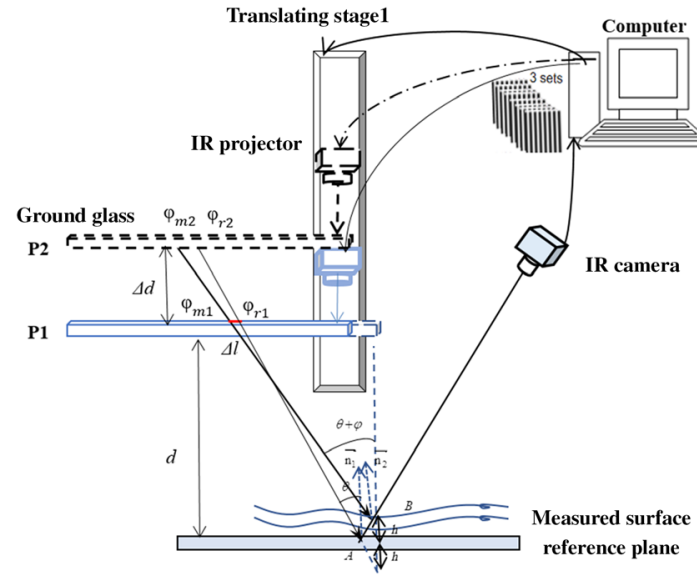

(a)

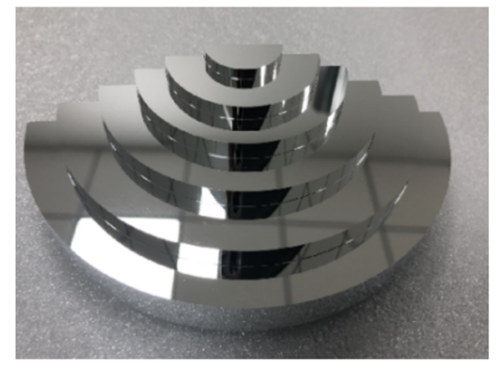

(c)

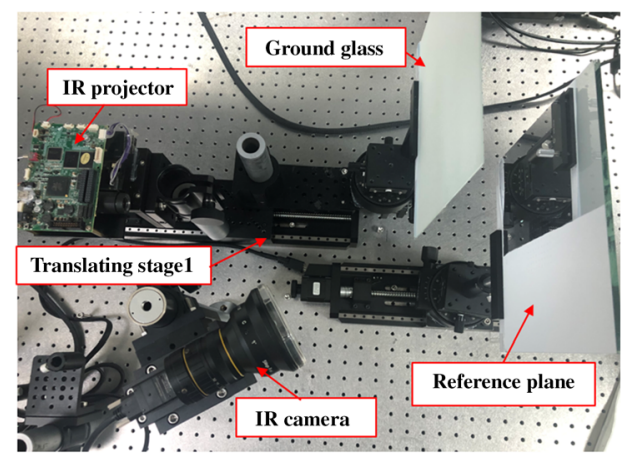

(b)

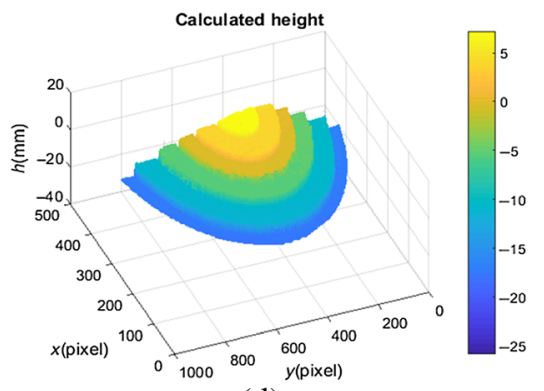

(d)

Fig. 6 Measurement example of the improved IR-PMD. ${ }^{66}$ (a) Principle of improved IR-PMD. (b) Hardware of the experimental setup. (c) Artificial fan-shaped step. (d) Measured depth of the fan-shaped step. 
Figures 6(a) and 6(b) are the schematic diagram and hardware compositions of the improved IR-PMD system, respectively. A smaller IR projector projects sinusoidal fringe patterns onto a ground glass, which is regarded as an IR digital screen. The ground glass and projector are moved to two different positions by an accurate translating stage during the measurement procedure to realize two screens design. From the reconstructed 3D shape of the artificial fan-shaped step shown in Figs. 6(c) and 6(d), it clearly shows that the proposed IR-PMD-based method can measure the specular objects having isolated and/or discontinuous surfaces. The effectiveness and accuracy of the proposed method were validated by the experiment on the artificial fanshaped mirror step. To test the repeatability of the presented system, measurement uncertainties of the improved IR-PMD ${ }^{67}$ method have been studied by evaluating the height value changes of an artificial steps 1 and 2, as shown in Fig. 7. Figure 7(a) is the measured depth of the artificial step. Figure 7(b) is the fitting results of the distance between 1 and 2 step surface. Figures 7(c) and 7(d) are the height value of one pixel at different measurement times and multiple pixels at one measurement time between steps 1 and 2, respectively. It can be seen that the maximum values of step height change are 0.013 and $0.015 \mathrm{~mm}$, the standard deviation values for the chosen pixels are 0.0038 and $0.004 \mathrm{~mm}$, respectively. These results verify the presented improved IR-PMD69 method has good repeatability of measurement.

By utilizing IR as a light source, this method can not only effectively reduce the influence of ambient light on the measurement system and measure the objects having isolated and/or discontinuous surfaces but also reduce the random errors caused by moving the measured objects and reference during the measurement procedure.

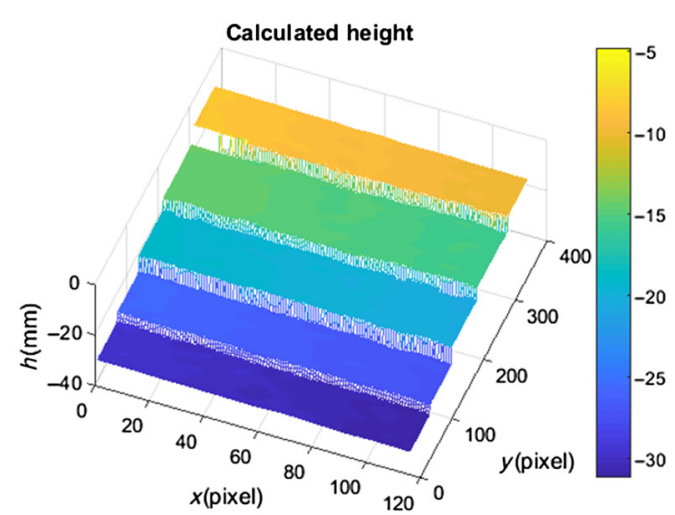

(a)

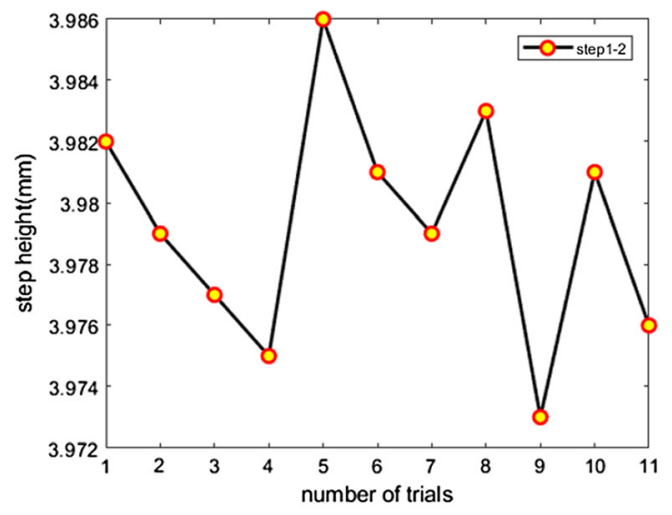

(c)

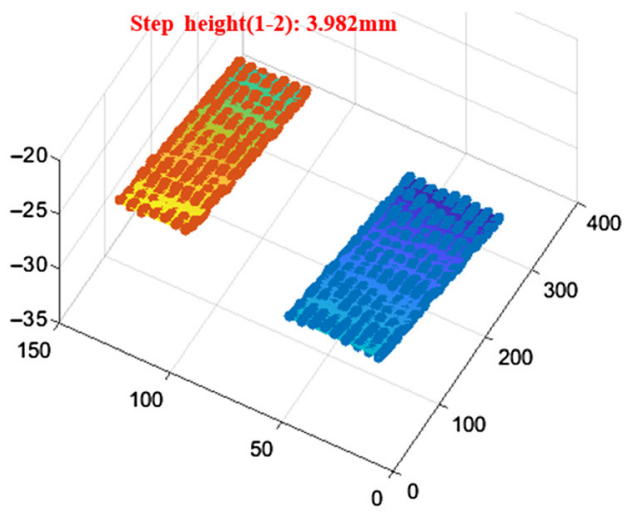

(b)

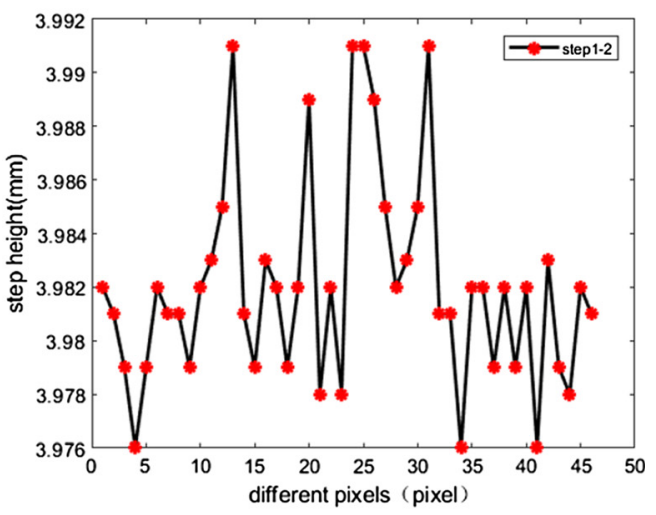

(d)

Fig. 7 Measurement uncertainties of the improved IR-PMD ${ }^{67}$ method by reconstructing an artificial step. (a) Measured depth of the artificial step. (b) Fitting results of the distances between 1 and 2 step surface. (c) Height value of one pixel at different measurement times between step 1 and 2 . (d) Height value of multiple pixels between step 1 and 2 . 


\subsection{Partial Reflective Surfaces}

In industrial fields, aerospace, and real life, some discontinuous components have both diffused and specular surface on the same object. How to reconstruct the 3D shape of these discontinuous diffused/specular reflective surfaces is a challenging problem. In order to overcome this problem, Liu et al. ${ }^{68}$ presented a method to measure the $3 \mathrm{D}$ shape of this kind of objects having isolated and/or discontinuous surfaces by combining FPP and DPMD, as shown in Fig. 8. Compared with DPMD, the proposed method adds a projector in the measurement system, and a diffused/specular reference plane is used to establish the geometric relationship. Similar to DPMD, the method works on simultaneously reconstructing both height and phase from the measured phases by establishing a mathematical model, instead of integrating the slopes. Then, after the geometric parameters $\Delta d, d$, and $a_{i}(u, v)$ calibrated, absolute phase data of the tested diffused/specular reflective surfaces will be calculated from the captured deformed fringes. Finally, height information of the discontinuous measured objects can be calculated according to Eq. (2):

$$
h=\left\{\begin{array}{ll}
\frac{d \cdot\left[\left(\varphi_{\mathrm{m} 2}-\varphi_{\mathrm{m} 1}\right)-\left(\varphi_{\mathrm{r} 2}-\varphi_{\mathrm{r} 1}\right)\right]-\Delta d \cdot\left(\varphi_{\mathrm{m} 1}-\varphi_{\mathrm{r} 1}\right)}{\left(\varphi_{\mathrm{r} 2}-\varphi_{\mathrm{r} 1}\right)+\left(\varphi_{\mathrm{m} 2}-\varphi_{\mathrm{m} 1}\right)} ; & \text { (specular surface) } \\
\sum_{i=0}^{n} a_{i}(\psi-\varphi)^{i} ; & \text { (diffused surface) }
\end{array},\right.
$$

where $\psi$ is the absolute phase of the deformed pattern, $\varphi$ is the reference phase obtained when the calibration board is at the reference position.

The projector and LCD screen project and display fringe patterns through red, green, and blue channels, simultaneously. Figure 8(a) shows the mathematical model of the proposed method. The hardware system is shown in Fig. 8(b). A composite plane calibration board having

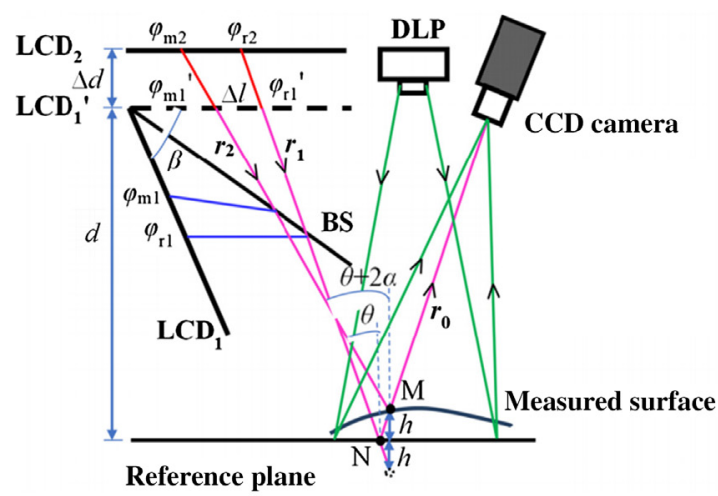

(a)

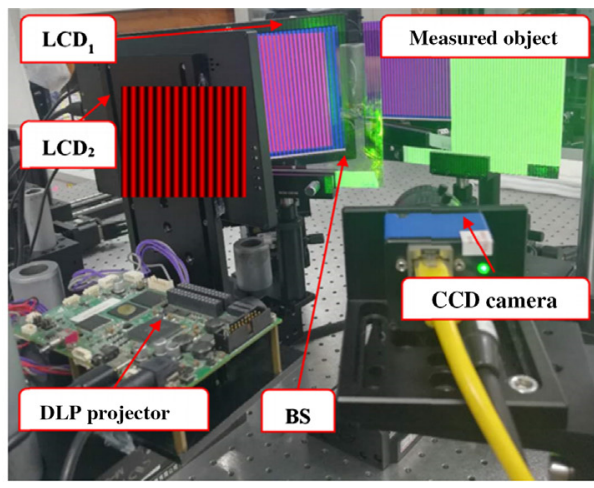

(b)

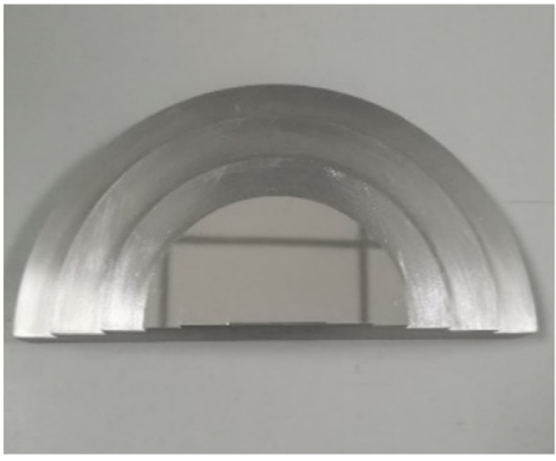

(c)

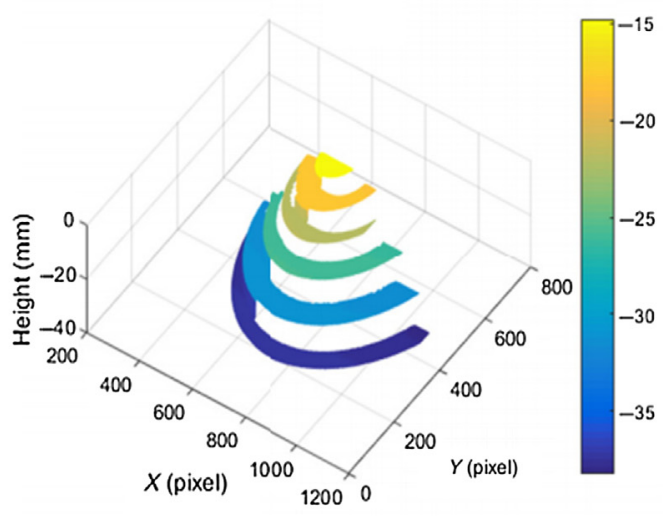

(d)

Fig. 8 Measurement principle of Liu et al.'s ${ }^{68}$ method by reconstructing partial reflective surfaces. (a) Mathematical model of the method. (b) Hardware of the experimental setup. (c) Artificial fanshaped diffused/specular reflective step. (d) Measured depth of the fan-shaped step. 
Zhang et al.: Phase measuring deflectometry for obtaining 3D shape of specular surface...

Table 1 Measurement results of the artificial fan-shaped standard step (unit: $\mathrm{mm}$ ).

\begin{tabular}{lccc}
\hline \hline Step plane & Actual step distance & Measured distance & Absolute error \\
\hline $1-2$ & 3.000 & 2.974 & 0.026 \\
$2-3$ & 4.000 & 4.023 & 0.023 \\
$3-4$ & 5.000 & 5.041 & 0.041 \\
$4-5$ & 5.500 & 5.518 & 0.018 \\
$5-6$ & 6.500 & 6.481 & 0.019 \\
\hline \hline
\end{tabular}

diffused/specular surface has been chosen as a reference. An artificial fan-shaped diffused/specular reflective step was measured using the proposed method, as shown in Fig. 8(c). Figure 8(d) is the reconstructed 3D shape of the artificial fan-shaped diffused/specular reflective step. To verify the measurement accuracy of this proposed system, distances between neighbor step surfaces of the diffused/specular reflective step have been calculated, as listed in Table 1. The maximum absolute error is $0.041 \mathrm{~mm}$. These results verify that the proposed method can measure discontinuous objects with diffused/specular reflective surfaces effectively and accurately.

In summary, all above methods have their advantages and disadvantages, as listed in Table 2. Advances of PMD techniques have made it possible to measure specular components having discontinuous and/or isolated surfaces with high precision. IR-PMD is a superior technique, which introduces IR light as a light source to reduce the effect of the ambient light. Moreover, a major advancement has been proposed in Liu et al.'s method, ${ }^{68}$ which can be used to measure partial reflective objects having discontinuous surfaces effectively and accurately.

Table 2 Advantages and disadvantages of various methods in PMD technology.

\begin{tabular}{|c|c|c|c|}
\hline Method & Ref & Advantage & Disadvantage \\
\hline Traditional PMD & 18,36 & High accuracy. & $\begin{array}{l}\text { (1) Error accumulation during } \\
\text { integration. } \\
\text { (2) Inability to measure discontinuous } \\
\text { specular surfaces. } \\
\text { (3) Inability to measure discontinuous } \\
\text { partial reflective surfaces. } \\
\text { (4) Effect of ambient light. }\end{array}$ \\
\hline DPMD & $49-51$ & $\begin{array}{l}\text { Ability to measure discontinuous } \\
\text { specular surfaces. }\end{array}$ & $\begin{array}{l}\text { (1) Effect of ambient light. } \\
\text { (2) Inability to measure discontinuous } \\
\text { partial reflective surfaces. }\end{array}$ \\
\hline IR-PMD & 66 & $\begin{array}{l}\text { (1) Ability to measure discontinuous } \\
\text { specular surfaces. } \\
\text { (2) High accuracy. Effect of ambient } \\
\text { light can be reduced. }\end{array}$ & $\begin{array}{l}\text { (1) Effect of random noise. } \\
\text { (2) Inability to measure discontinuous } \\
\text { partial reflective surfaces. }\end{array}$ \\
\hline Improved IR-PMD & 67 & $\begin{array}{l}\text { (1) Ability to measure discontinuous } \\
\text { specular surfaces. } \\
\text { (2) High accuracy. Effects } \\
\text { of ambient light and random } \\
\text { noise can be reduced. }\end{array}$ & $\begin{array}{l}\text { Inability to measure discontinuous } \\
\text { partial reflective surfaces. }\end{array}$ \\
\hline Liu et al.'s method & 68 & $\begin{array}{l}\text { Ability to measure discontinuous } \\
\text { partial reflective surfaces. }\end{array}$ & Effect of ambient light. \\
\hline
\end{tabular}


The recent advancements on PMD mentioned above show that accurately reconstructing discontinuous specular surfaces becomes possible.

\section{Error Sources Analysis}

To obtain 3D shape data of specular surfaces accurately, it is important and necessary to analyze the error sources in the PMD systems, including geometric calibration error, phase error (which caused by the nonlinear influence of imaging and projecting system, display screen, random phase error, and sampling phase error), on the measurement results and to evaluate the performance of the $3 \mathrm{D}$ shape measurement system.

\subsection{Geometric Calibration Error}

PMD system is based on the principle of geometrical optics, so the relative positions of each component in the system must be obtained accurately before measurement. Therefore, geometric calibration is and will always be a critical procedure to determine the relationship between phase and depth (or slope) of the measured specular surfaces. The traditional methods realize the calibration process with the help of calibration tools, ${ }^{69,70}$ yet errors may exist in the additional calibration tools. To overcome this shortcoming, self-calibration methods are studied to utilize the displaying screen to calibrate PMD system. ${ }^{71}$ However, a pinhole camera model is used in the imaging system, which is far from a true camera model. Therefore, accurate geometric calibration of the system remains a challenge to the PMD system.

\subsection{Phase Error}

The measurement principle of PMD is based on phase calculation. Therefore, the phase error is a significant error source for a PMD system. Phase error sources mainly arise from nonlinear response, lens distortion of imaging and projecting system, random error and sampling error, etc. Since the slope and depth data are calculated from the phase information, the calculation process is affected by the phase error in the PMD system. Therefore, it is necessary to analyze the sources of phase error and reduce the influence of phase error.

\subsubsection{Nonlinear response and lens distortion of display and imaging components}

Due to the gamma effect of the display and imaging components, the system's light intensity has a nonlinear response, which makes the standard shape of sinusoidal fringe patterns into nonsinusoidal shapes, resulting in phase errors. Moreover, the performance of the PMD systems is also affected by lens distortion. It is critical to remove the errors brought by nonlinear response and lens distortion of the display and imaging components. Many methods have been proposed to compensate for nonlinear response. ${ }^{72,73}$ For correcting lens distortions, Guo et al. ${ }^{74}$ proposed a pre- and a postcompensation method for calibrating the measurement system. Yang et al. ${ }^{75}$ presented a projector calibration method, which can fully represent the lens distortion using a directly measured distortion map, instead of using an approximate model in existing methods. Software-based technique has been used to compensate for nonlinear response and lens distortion. ${ }^{73}$ The nonlinear response and lens distortion are corrected by generating a sinusoidal fringe pattern with the software.

\subsubsection{Display screen}

Since an LCD screen is a necessary component of most PMD systems, the imperfect performance of the screen affects the measurement accuracy as well. The error sources that affect 3D shape measurement results mainly include: the flatness variation of the display surface, the refraction effects in the transparent layers, and grayscale and color characteristic problems. ${ }^{76}$ Most researchers have assumed the LCD screen as an ideal plane for phase calculations in 
PMD systems. However, in an actual situation, the manufactured screen is not flat. The fringe patterns displayed on the screen will be deformed due to the imperfect performance of the screen, which will affect the measurement accuracy of the PMD. Therefore, the actual surface geometry of the LCD screen needs to be determined for more accurate measurement system. Furthermore, the refraction effect in the transparent layers of the LCD screen is a major contributor to the system measurement error. To improve the calibration accuracy of the fringe projection system, Chen et al. ${ }^{77}$ proposed a camera calibration method using an LCD display as the calibration board. In this method, the phase error introduced by the glass plate of the LCD screen is considered and corrected. The refraction error model of LCD is also applicable to PMD systems. Li et al. ${ }^{41}$ proposed a phase measurement deflectometry incorporating with a refraction model to measure mirror surface. Because of the light propagation path is accurately determined by introducing the refraction model, the system measurement accuracy can be improved.

\subsubsection{Random and sampling phase error}

Since visible light is sensitive to the ambient light, the reconstructed 3D shapes of PMD systems will be affected by the external environment in actual measurements. Moreover, an important factor that causes measurement accuracy is the random and sampling phase error. Because continuous sinusoidal fringe patterns are sampled by the screen's pixels during displaying images, the random and sampling error introduces obvious phase error. To obtain more accurate 3D shape of specular surfaces, compensation methods of random and sampling phase error need to be studied in future research.

\section{Challenges and Further Developments}

Although a lot of research has been done on the specular object measurement methods based on the full-field fringe reflection, some deficiencies are still remaining in PMD and more robust and flexible solutions are required.

\subsection{Complex Surfaces with Large Curvature}

Some specular components have large curvature changing surfaces. It is a challenging problem to measure the 3D shape of these kinds of objects with a high accuracy using the existing PMD systems. The factors that affect its accuracy of the large curvature measurement are as follows: (1) the reflected ray through the measured large curvature changing surface may exceed the physical range of the display screen; (2) the large curvature may blur the reflected fringe patterns and increase the phase error. Few people have devoted to reconstructing the 3D data of specular surfaces with large curvature. Craves et al. ${ }^{78}$ proposed a method using a conical mirror to measure roundness of a cylindrical surface so that the cylindrical surface can be transferred into a planar image. In order to further measure specular surfaces with high curvature, curved screens can be introduced into PMD systems.

\subsection{High Accuracy}

As mentioned in Sec. 4, there are many factors that affect the accuracy of the existing PMD systems. In order to further improve the measurement accuracy of PMD systems, it is necessary to compensate for those errors introduced by different error sources.

\subsection{Fast Measurement}

In actual production, there are a large number of specular objects that need to be measured in real-time acquisition and analyzed, which puts forward fast requirements for optical measurement methods. Fast measurement is usually considered to improve hardware efficiency and reduce shooting patterns. Trumper et al. ${ }^{79}$ simultaneously encoded three sets of orthogonal sinusoidal fringe patterns into red, green, and blue channels to reduce the acquisition time to one-third 
and can be used to measure dynamic objects. However, this method will arise in color fringe patterns, such as crosstalk and chromatic aberration between color channels.

\subsection{Portable}

The existing PMD systems have disadvantages of heavy and inconvenient to assemble together, carry around, and install in various environments. Therefore, miniaturization and portability are two of the important development trends of PMD systems. It is a good choice to embed a PMD system into a manufacturing system to improve measurement accuracy. Butel et al ${ }^{80}$ established a portable PMD system based on mobile devices, which can run on any mobile device with a front camera and quickly complete 3D shape measurement within 1 min. Maldonado et al. ${ }^{81}$ developed a new portable slope-measuring portable optical test system, which can achieve a better surface accuracy and slope precision.

\section{Conclusions}

Recent advancements of PMD for obtaining 3D shape of specular surface are reviewed in this paper. It mainly introduces the measurement principles of PMD systems, the advancements of PMD, error sources analysis, challenges, and further developments. Although some aspects of the PMD technique have been studied to measure discontinuous and/or specular surfaces, there are still many unsolved challenges in measuring their 3D shape accurately and quickly. Therefore, further studies are still necessary to make the PMD technique more flexible to calibrate, more portable to use, more accurate, and quicker in actual measurements for industrial manufacturing and aerospace applications.

\section{Acknowledgments}

The authors wish to acknowledge the support by the National Key R\&D Program of China (under Grant No. 2017YFF0106404) and the National Natural Science Foundation of China (under Grant No. 51675160, 52075147).

\section{References}

1. Z. Zhang, "Review of single-shot 3D shape measurement by phase calculation-based fringe projection techniques," Opt. Lasers Eng. 50, 1097-1106 (2012).

2. C. Chen et al., "Generic exponential fringe model for alleviating phase error in phase measuring profilometry," Opt. Lasers Eng. 110, 179-185 (2018).

3. C. Zuo et al., "High-speed three-dimensional profilometry for multiple objects with complex shapes," Opt. Express 20(17), 19493-19510 (2012).

4. G. Brown, "Overview of three-dimensional shape measurement using optical methods," Opt. Eng. 39(1), 10-22 (2000).

5. Z. Wang et al., "Single-shot 3D shape measurement of discontinuous objects based on coaxial fringe projection system," Appl. Opt. 58(5), A169-A178 (2019).

6. S. Zhang, "Recent progresses on real-time 3D shape measurement using digital fringe projection techniques," Opt. Lasers Eng. 48, 149-158 (2010).

7. X. Bai et al., "Person recognition using 3-D palmprint data based on full-field sinusoidal fringe projection," IEEE Trans. Instrum. Meas. 68(9), 3287-3298 (2019).

8. S. Zhang, "High-speed 3D shape measurement with structured light methods: a review," Opt. Lasers Eng. 106, 119-131 (2018).

9. B. Li et al., "Computer-aided-design-model-assisted absolute three-dimensional shape measurement," Opt. Express 56(24), 6770-6776 (2017).

10. S. Jeught et al., "Real-time structured light profilometry: a review," Opt. Laser Eng. 87, 18-31 (2016).

11. J. Xu et al., "3D multi-directional sensor with pyramid mirror and structured light," Opt. Lasers Eng. 93, 156-163 (2017). 
Zhang et al.: Phase measuring deflectometry for obtaining 3D shape of specular surface...

12. P. David et al., "Effect of matte coating on 3D optical measurement accuracy," Opt. Mater. 40, 1-9 (2015).

13. B. Wang et al., "Moire deflectometry based on Fourier-transform analysis," Measurement 25(4), 249-253 (1999).

14. G. Mansour, "A developed algorithm for simulation of blades to reduce the measurement points and time on coordinate measuring machine (CMM)," Measurement 54, 51-57 (2014).

15. J. Wyant et al., "Recent advances in interferometric optical testing," Laser Focus/Electrooptics 21(11), 118-132 (1985).

16. S. Ettl et al., "Shape reconstruction from gradient data," Appl. Opt. 47(12), 2091-2097 (2008).

17. Z. Niu et al., "3D shape measurement of discontinuous specular objects based on advanced PMD with bi-telecentric lens," Opt. Express 26(2), 1615 (2018).

18. Y. Liu et al., "Fast and accurate deflectometry with crossed fringes," Adv. Opt Techn. 3(4), 41-45 (2017).

19. K. Mitsui et al., "Development of a high resolution sensor for surface roughness," Opt. Eng. 27(6), 498-502 (1988).

20. X. Jiang et al., "Fast surface measurement using wavelength scanning interferometry with compensation of environmental noise," Appl. Opt. 49, 2903-2909 (2010).

21. P. Murphy et al., "Stitching interferometry: a flexible solution for surface metrology," Opt. Photonics News 14(5), 38-43 (2004).

22. A. Dávila, "Wavelength scanning interferometry using multiple light sources," Opt. Express 24(5), 5311-5322 (2016).

23. G. Häusler et al., "Microdeflectometry-a novel tool to acquire three-dimensional microtopography with nanometer height resolution," Opt. Lett. 33, 396-398 (2008).

24. Y. Liu et al., "High-accuracy measurement for small scale specular objects based on PMD with illuminated film," Opt. Lasers Eng. 44(2), 459-462 (2012).

25. C. Guo et al., "Improved phase-measuring deflectometry for aspheric surfaces test," Appl. Opt. 55(8), 2059-2064 (2016).

26. M. Knauer et al., "Phase measuring deflectometry: a new approach to measure specular freeform surfaces," Proc. SPIE 5457, 366-376 (2004).

27. Z. Zhang et al., "Three-dimensional shape measurements of specular objects using phasemeasuring deflectometry," Sensors 17(12), 2835 (2017).

28. C. Li et al., "Posed relationship calibration with parallel mirror reflection for stereo deflectometry," Opt. Eng. 57(3), 034103 (2018).

29. T. Oiwa, "Coordinate measuring machine using parallel mechanism," in Proc. 16th IMEKO World Congr. Vol. 8, pp. 211-214 (2000).

30. M. Cedilnik, et al., "Scanning errors identification using touch trigger probe head," Int. J. Comput. Mater. Sci. Surf. Eng. 1(3), 275-291 (2007).

31. M. Mahboubkhah et al., "An investigation on measurement accuracy of digitizing methods in turbine blade reverse engineering," Proc. Inst. Mech. Eng., Part B: J. Eng. Manuf. 232(9), 1653-1671 (2018).

32. M. Aliakbari et al., "An adaptive computer-aided path planning to eliminate errors of contact probes on free-form surfaces using a 4-DOF parallel robot CMM and a turn-table," Measurement 166, 108216 (2020).

33. H. Muhamedsalih et al., "Accelerated surface measurement using wavelength scanning interferometer with compensation of environmental noise," Procedia CIRP 10, 70-76 (2013).

34. Z. Zhang et al., "Recent advance on phase measuring deflectometry for obtaining 3D shape of specular surface," Proc. SPIE 11552, $115520 \mathrm{Z}$ (2020).

35. A. Agrawal et al., "What is the range of surface reconstructions from a gradient field," Lect. Notes Comput. Sci. 3951, 578-591 (2006).

36. L. Huang et al., "Comparison of two-dimensional integration methods for shape reconstruction from gradient data," Opt. Lasers Eng. 64, 1-11 (2015).

37. L. Huang et al., "Shape reconstruction from gradient data in an arbitrarily-shaped aperture by iterative discrete cosine transforms in South well configuration," Opt. Lasers Eng. 67, 176-181 (2015). 
38. H. Guo et al., "Specular surface measurement by using a moving diffusive structured light source," Proc. SPIE 6834, 68343E (2007).

39. H. Guo et al., "Specular surface measurement by using least squares light tracking technique," Opt. Lasers Eng. 48, 166-171 (2010).

40. M. Petz et al., "Measurement of optically effective surfaces by imaging of gratings," Proc. SPIE 5144, 288-294 (2003).

41. C. Li et al., "Phase measurement deflectometry with refraction model and its calibration," Opt. Express 26(26), 33510-33522 (2018).

42. M. Petz et al., "Reflection grating method for 3D measurement of reflecting surfaces," Proc. SPIE 4399, 35-41 (2001).

43. Y. Xiao et al., "Three-dimensional shape measurement of aspheric mirrors with fringe reflection photogrammetry," Appl. Opt. 51(4), 457-464 (2012).

44. Y. Tang et al., "3D shape measurement of the aspheric mirror by advanced phase measuring deflectometry," Opt. Express 16, 15090-15096 (2008).

45. Y. Xiao et al., "Optical fringe-reflection deflectometry with bundle adjustment," Opt. Lasers Eng. 105, 132-140 (2018).

46. H. Ren et al., "Iterative optimization calibration method for stereo deflectometry," Opt. Express 23(17), 22060-22068 (2015).

47. H. Ren et al., "Improvement of high-order least-squares integration method for stereo deflectometry," Appl. Opt. 54(34), 10249 (2015).

48. Y. Xu et al., "Enhancement of measurement accuracy of optical stereo deflectometry based on imaging model analysis," Opt. Lasers Eng. 111, 1-7 (2018).

49. Y. Liu et al., "Full-field 3D shape measurement of discontinuous specular objects by direct phase measuring deflectometry," Sci. Rep. 7, 10293 (2017).

50. P. Zhao et al., "Performance analysis and evaluation of direct phase measuring deflectometry," Opt. Lasers Eng. 103, 24-33 (2018).

51. S. Huang et al., "Distance calibration between reference plane and screen in direct phase measuring deflectometry," Sensors 18(1), 144 (2018).

52. Y. Xu et al., "A brief review of the technological advancements of phase measuring deflectometry," PhotoniX 1(1), 14 (2020)

53. M. Takeda et al., "Fourier transform profilometry for the automatic measurement of 3-D object shapes," Appl. Opt. 22(24), 3977-3982 (1983).

54. P. Tavares et al., "Orthogonal projection technique for resolution enhancement of the Fourier transform fringe analysis method," Opt. Commun. 266, 465-468 (2006).

55. S. Feng et al., "A carrier removal technique for Fourier transform profilometry based on principal component analysis," Opt. Lasers Eng. 74, 80-86 (2015).

56. V. Srinivasan et al., "Automated phase-measuring profilometry of 3-D diffuse objects," Appl. Opt. 23(18), 3105 (1984).

57. Y. Surrel, "Design of algorithms for phase measurements by the use of phase stepping," Appl. Opt. 35(1), 51-60 (1996).

58. K. Qian, "Two-dimensional windowed Fourier transform for fringe pattern analysis: principles, applications and implementations," Opt. Lasers Eng. 45, 304-317 (2007).

59. L. Huang et al., "Fast full-field out-of-plane deformation measurement using fringe reflectometry," Opt. Lasers Eng. 50, 529-533 (2012).

60. X. Su et al., "Fourier transform profilometry: a review," Opt. Lasers Eng. 35, 263-284 (2001).

61. W. Chen et al., "Method for eliminating zero spectrum in Fourier transform profilometry," Opt. Lasers Eng. 43, 1267-1276 (2005).

62. Z. Zhang et al., "Comparison of Fourier transform, windowed Fourier transform, and wavelet transform methods for phase calculation at discontinuities in fringe projection prolometry," Opt. Lasers Eng. 50, 1152-1160 (2012).

63. X. Su et al., "Reliability-guided phase unwrapping algorithm: a review," Opt. Lasers Eng. 42, 245-261 (2004).

64. M. Zhao et al., "Quality-guided phase unwrapping technique: comparison of quality maps and guiding strategies," Appl. Opt. 50, 6214-6224 (2011). 
Zhang et al.: Phase measuring deflectometry for obtaining 3D shape of specular surface...

65. C. Zuo et al., "Temporal phase unwrapping algorithms for fringe projection profilometry: a comparative review," Opt. Lasers Eng. 85, 84-103 (2016).

66. C. Chang et al., "Measurement of the three-dimensional shape of discontinuous specular objects using infrared phase-measuring deflectometry," Sensors 19(21), 4621 (2019).

67. C. Chang et al., "Improved infrared phase measuring deflectometry method for the measurement of discontinuous specular objects," Opt. Lasers Eng. 134, 106194 (2020).

68. X. Liu et al., "3D shape measurement of diffused/specular surface by combining fringe projection and direct phase measuring deflectometry," Opt. Express 28(19), 27561-27574 (2020).

69. Y. Xu et al., "An iterative distortion compensation algorithm for camera calibration based on phase target," Sensors 17(6), 1188 (2017).

70. L. Huang et al., "Modal phase measuring deflectometry," Opt. Express 24, 24649-24664 (2016).

71. Y. Xu et al., "A holistic calibration method with iterative distortion compensation for stereo deflectometry," Opt. Lasers Eng. 106, 111-118 (2018).

72. S. Lei et al., "Flexible 3-D shape measurement using projector defocusing," Opt. Lett. 34, 3080-3082 (2009).

73. Z. Zhang et al., "Time efficient color fringe projection system for 3D shape and color using optimum 3-frequency selection," Opt. Express 14, 6444-6455 (2006).

74. H. Guo et al., "Iterative calibration method for measurement system having lens distortions in fringe projection profilometry," Opt. Express 28(2), 1177 (2019).

75. S. Yang et al., "Flexible digital projector calibration method based on per-pixel distortion measurement and correction," Opt. Lasers Eng. 92(5), 29-38 (2017).

76. M. Petz et al., "Systematic errors in deflectometry induced by use of liquid crystal displays as reference structure," in Proc. 21st IMEKO TC2 Symp. Photon. Meas., pp. 16-18 (2013).

77. C. Chen et al., "High accuracy 3D calibration method of phase calculation-based fringe projection system by using LCD screen considering refraction error," Opt. Lasers Eng. 126, 105870 (2020).

78. L. Graves et al., "Infinite deflectometry enabling $2 \pi$-steradian measurement range," Opt. Express 27(5), 7602-7615 (2019).

79. I. Trumper et al., "Instantaneous phase shifting deflectometry," Opt. Express 24(24), 27993-28007 (2016).

80. G. Butle et al., "Deflectometry using portable devices," Opt. Eng. 54(2), 025111 (2015).

81. A. Maldonado et al., "Development of a portable deflectometry system for high spatial resolution surface measurements," Appl. Opt. 53(18), $4023-4036$ (2014).

Zonghua Zhang is a professor at Hebei University of Technology, China. He received his $\mathrm{PhD}$ from Tianjin University, China. He worked in Ruhr University Bochum of Germany, Queen's University of Canada, Heriot-Watt University, University of Leeds, and University of Huddersfield of United Kingdom. His research interests are 3D optical measurement, fringe projection profilometry, and phase measuring deflectometry. He has published more than 180 papers. From 2016 to 2018, he was an EU Marie Curie Fellow in University of Huddersfield. He is an associate editor for Optics Express.

Caixia Chang is a PhD candidate in the School of Mechanical Engineering, Hebei University of Technology, China. Her current research focuses on three-dimensional optical measurement and machine vision.

Xiaohong Liu is a PhD candidate in the School of Mechanical Engineering, Hebei University of Technology, China. Her current research focuses on three-dimensional optical measurement and machine vision.

Ziyu Li is a PhD candidate in the School of Mechanical Engineering, Hebei University of Technology, China. Her current research focuses on three-dimensional optical measurement and machine vision. 
Yanqing Shi is a master in the School of Mechanical Engineering, Hebei University of Technology, China. His current research focuses on three-dimensional optical measurement and machine vision.

Nan Gao received his BS, MS, and $\mathrm{PhD}$ degrees in biomedical engineering from Tianjin University, Tianjin, China, in 2006, 2008, and 2012, respectively. Since 2012, he has been a lecturer and associate professor with the School of Mechanical Engineering, Hebei University of Technology. He is the author of more than 30 articles. His research interests include threedimensional measurement of structured light projection, infrared absorption spectrum detection, machine vision, and image processing

Zhaozong Meng received his $\mathrm{PhD}$ in computer science from the University of Huddersfield, West Yorkshire, United Kingdom, in 2014. Since 2018, he has been a lecturer with the School of Mechanical Engineering, Hebei University of Technology, Tianjin, China. Since 2020, he has been promoted as an associate professor. His research interests include advanced sensor techniques, wearable devices and body area network, manufacturing intelligence, industrial IoT, and cyberphysical systems 